\title{
An Ingestible Self-Polymerizing System for Targeted Sampling of Gut Microbiota and Biomarkers
}

\author{
(Supplementary Information)
}

Lu Chen ${ }^{1}$, Lina Gruzinskyte1,2, Steffen Lynge Jørgensen³ ${ }^{3}$ Anja Boisen ${ }^{1}$

Sarvesh Kumar Srivastava ${ }^{1 *}$

\begin{abstract}
1The Danish National Research Foundation and Villum Foundation's Center for Intelligent Drug Delivery and Sensing Using Microcontainers and Nanomechanics (IDUN), Department of Health Technology, Technical University of Denmark, 2800

Kgs. Lyngby, Denmark

${ }^{2}$ Department of Pharmacy, Faculty of Health and Medical Sciences, University of Copenhagen, Universitetsparken 2, 2100 København Ø Denmark ${ }^{3}$ Danish Meat Research Institute, Danish Technological Institute, Gregersensvej, 2630 Taastrup Denmark
\end{abstract}

E-mail: sksr@dtu.dk

\section{Materials:}

SU-8 photoresist (MicroChem), silicone elastomer curing agent, silicone elastomer, and rhodamine $6 \mathrm{G}$ (R6G), $1 \mathrm{M} \mathrm{NaOH}$ solution, $1 \mathrm{M} \mathrm{HCl}$ solution. Ascorbic acid, Iron(III) chloride ( $\mathrm{FeCl} 3$ ), Poly(ethylene glycol) diacrylate (PEGDA) monomer m.w. 575. Fluorescein isothiocyanate (FITC)-insulin.

Human colorectal adenocarcinoma cells Caco-2 and HT29-MTX-E12. Dulbecco's modified eagle medium (DMEM), fetal bovine serum (FBS), penicillin-streptomycin (P/S), non-essential amino acids (NEAA), 4\% (w/v) fresh paraformaldehyde (PFA), 0.05\% Saponin/1\%BSA/PBS, 3\% bovine serum albumin (BSA), Hoechst33342, phosphate buffered saline (PBS), trypsin/EDTA (0.05\%). Transwell plates containing 
porous polyester membrane inserts $\left(1.12 \mathrm{~cm}^{2}, 0.4 \mathrm{~mm}\right.$ pores $)$. PierceTM Protease Assay Kit. Eudragitß L 100 and Torpak® Size 9 gelatin capsules.

\section{Methods}

\section{Fabrication of TRAP microdevices}

The conventional epoxy based negative photoresist SU-8 (MicroChem, USA) was used to fabricate the microcontainers (hollow cylindrical structures) using a two-step photolithography technique. The first layer of SU-8 $(2035,69.95 \mathrm{wt} \%)$ was spin coated (Süss MicroTec RCD8 with Gyrset) on a fluorocarbon coated silicon wafer and soft baked at $50{ }^{\circ} \mathrm{C}$ for $2 \mathrm{~h}$ (temperature ramping at a rate of $2{ }^{\circ} \mathrm{C} / \mathrm{min}$ ) and slowly cooled down to room temperature. The bottom of the microcontainers (thickness $35 \mu \mathrm{m}$ ) was defined using UV exposure for $30 \mathrm{sec}$ using soft contact (Süss Mask Aligner MA6, equipped with in-line notch filter) as well as two bursts of $250 \mathrm{~mJ} / \mathrm{cm}^{2}$. A post exposure baking was conducted at $50{ }^{\circ} \mathrm{C}$ for $6 \mathrm{~h}$ (with the same ramping conditions as mentioned before). The second layer of resist (SU-8 2075, 73.45 wt\%) was spin coated and prebaked at $50{ }^{\circ} \mathrm{C}$ for $10 \mathrm{~h}$ to define the microwell wall (height $220 \mu \mathrm{m}$ ) using the same temperature ramping condition. A global WEC chuck was used to conduct the UV exposure in proximity mode, followed by two bursts of $250 \mathrm{~mJ} / \mathrm{cm}^{2}$ for $30 \mathrm{sec}$, after which, a post exposure baking was performed at $50{ }^{\circ} \mathrm{C}$ for another $10 \mathrm{~h}$ using the same temperature ramping condition. The resulting substrate was developed in mr-Dev 600 for $2 \times 20$ minutes. Then the resist on the substrate was flushed with isopropanol and left to dry before the inspection. The silicon wafer was cleaved into several rectangular slides, each containing 625 microcontainers. The height and inner diameter of the resulting microcontainers were $220 \mu \mathrm{m}$ and $190 \mu \mathrm{m}$ respectively, with a $25 \mu \mathrm{m}$ thick sealed bottom. Diced chip was teared off and placed in a sample holder. A very thin layer of PDMS mixture acting as a negative mask (PDMS monomers mixed with Iguracure D2959 at the volume ratio of 10:1) was spread across the spaces between the microwells,. Then PDMS was fully cured in the oven at $37^{\circ} \mathrm{C}$ overnight.

\section{Loading of TRAP microdevices}

Ascorbic acid, $\mathrm{FeCl}_{3}$ (III) and PEGDA monomer (Mn: 575) were purchased from Sigma-Aldrich, Denmark and were used with no pre-treatment. Ascorbic acid and $\mathrm{FeCl}_{3}$ (III) serving as redox initiators, were mixed uniformly in a weight ratio of 5:1 (wt./wt.) via a mortar and pestle and stored in a vacuum chamber at room temperature. A modified brush loading method was used to load the resulting reactant mixture into 
the TRAP bots (with PDMS negative mask). Then a thin layer of PEGDA monomer ( $20 \mu \mathrm{L}$ ) was evenly spread over the microcontainers using doctor blading technique. The resulting TRAP bots was dried and kept in a dry environment. Lastly, the negative PDMS mask was peeled off followed by the characterization and in vitro and in vivo studies.

Rhodamine 6G dye (R6G) (Sigma-Aldrich, Danmark) was used without any pretreatment to stain PEGDA hydrogel for easy visualization (it is not part of the standard reaction mixture). R6G was distributed in radical initiators at a weight ratio of 1:50 (wt./wt.) using a mortar and pestle. The resulting mixtures were loaded in TRAP bots, covered with a layer of PEGDA monomer via the same methods described above. The assembled bots were dried at $40{ }^{\circ} \mathrm{C}$ for 24 hours on a hot plate. A Carl Zeiss optical microscope Scope A1 coupled with brightfield, darkfield and C-DIC mode was used to visualize TRAP bots activation. Video $\mathbf{S} 1$ shows homogenous activation of multiple TRAP microdevices with R6G as a visual indicator (25 fps; speed 2X)

Fluorescein isothiocyanate (FITC)-insulin was mixed with reactant agents at a weight ratio of 1:50 and loaded into microcontainers to fabricate the TRAP bots using the same method as was used to prepare R6G containing TRAP bots. The chip with FITCinsulin TRAP bots was put in a plastic dish and wrapped with tinfoil. 20 min UV irradiation was used to sterilize the TRAP bots prior to cell studies.

\section{In vitro TRAP bots cell study}

\section{Cell culture}

Caco-2 and HT29-MTX-E12 cells were cultured in $75 \mathrm{~mm}^{2}$ tissue culture flasks in Dulbecco's Modified Eagle Medium containing $4.5 \mathrm{~g} / \mathrm{L}$ glucose (Sigma, Denmark) supplemented with $10 \%(\mathrm{v} / \mathrm{v})$ heat-inactivated fetal bovine serum (FBS; Sigma, Denmark), 1\% (v/v) nonessential amino acids (NEAA; Gibco, Denmark) and 1\% (v/v) penicillin-streptomycin (P/S; Gibco, Denmark). Cells were maintained at $37^{\circ} \mathrm{C}$ and $5 \%$ $\mathrm{CO}_{2}$ levels in a humidifying incubator. Cells were subcultured once they reached $70-80 \%$ confluence using trypsin/ETDA solution (0.05\%; Sigma, Denmark). All cells used for experiments were between passages 8-40.

\section{Co-culture of Caco-2/HT29-MTX-E12 (7:3) cells}

Co-culture of Caco-2/HT29-MTX-E12 cells were used for cell trap studies. Cells were seeded at a density of $1.5 \times 10^{5}$ cells $/ \mathrm{cm}^{2}$ at a ratio of $7: 3$. Cells were seeded onto polycarbonate 12-well Transwell filters (Corning Incorporated, USA; $0.4 \mu \mathrm{m}$ pore size, $1.12 \mathrm{~cm}^{2}$ surface area) and in the 4-well plate. Co-culture was maintained under 
standard incubation conditions for 21 days in Transwell inserts and 7 days in 4-well plate, and medium was changed every other day.

\section{Cell trapping studies}

Cell trapping study was performed on a co-culture after 7 days. The media was removed from the wells and 7 TRAP bots were placed on top of the cell layer. $200 \mu \mathrm{L}$ of fresh medium was added to each well and incubated for 4 hours.

\section{Morphological studies}

To visualize the cells, F-actin and nuclei were stained in cell monolayer that was fixed in $4 \%(\mathrm{v} / \mathrm{v}$ ) paraformaldehyde (Sigma, Denmark) for $30 \mathrm{~min}$, permeabilized with $0.05 \%$ Saponin/1\%BSA (Sigma, Denmark) for $30 \mathrm{~min}$ and blocked in 3\% bovine serum albumin (BSA; Sigma, Denmark) in phosphate buffer saline (PBS; Sigma, Denmark) for $1 \mathrm{~h}$. Staining of the nucleus and actin was carried out by diluting FITC-phalloidin (AlexaFluor 488; Invitrogen, Denmark) and Hoechst 33342 (Invitrogen, Denmark) in 1\% (v/v) BSA/PBS solution and incubated with the cells for $20 \mathrm{~min}$ and $10 \mathrm{~min}$ respectively at room temperature in the dark. Between each processing step, the cells were rinsed with PBS thrice.

Fluorescent confocal images of the cells were obtained using a confocal microscope (Carl Zeiss, Germany) and images were treated with Image J.

\section{Characterization}

Scanning electron microscopic pictures were taken with TM3030Plus Tabletop Microscope (Hitachi, Krefeld, Germany) with a voltage of $15 \mathrm{keV}$ and sputtered with a $15 \mathrm{~nm}$ gold layer. Attenuated Total Reflectance Fourier transform infrared spectra (ATR-FTIR) were recorded by a Perkin Elmer Spectrum at a resolution of $4 \mathrm{~cm}^{-1}$. Pristine TRAP microdevices were used for background measurement. Subsequent measurements were made at a low scan rate (5) to account for real-time polymerization.

\section{In vivo rat study}

TRAP microdevices were loaded into a Torpac $®$ size 9 gelatin capsule which was coated in a $12 \%(\mathrm{w} / \mathrm{v})$ Eudragit L100 solution in IPA with dibutyl sebacate as plasticizer in a $5 \% \mathrm{w} / \mathrm{w}$ ratio relative to Eudragit. Each size 9 capsule has c.a. 600 microdevices. Male Sprague-Dawley rats (Janvier Labs, Le Genest-Saint-Isle, France) were housed in groups of six per cage and allowed to acclimatize for at least one week with a reversed $12 / 12 \mathrm{~h}$ day/night cycle. Fasting of the rats was initiated $12-16 \mathrm{~h}$ prior to the 
studies and the study was conducted under full anesthesia. The abdominal cavity was opened after which an enteric-coated capsule with a tiny permanent magnet (size: 0.2 $\mathrm{cm} \times 0.1 \mathrm{~cm} ; 1.3 \mathrm{~T}$ ) inside is administered to the stomach using a gavage dosing tube. The capsule was dragged to the duodenum using an external NdFeB magnet (1.3 T). It must be noted that the role of magnet was only to drag the enteric-coated capsule across the stomach into the duodenum, after which, the capsule disintegrated and microdevices were released in the small intestine.

The rats $(n=2)$ were kept anesthetized for $3 \mathrm{~h}$, after which, they were euthanized by an intracardiac injection of pentobarbital $(100 \mathrm{mg} / \mathrm{kg})$. After euthanasia, the stomachs and small intestines were removed from two of the rats that had been administered TRAPs in order to localize their position and orientation (stored at $-20^{\circ} \mathrm{C}$ ). Note that microbiota analysis was made with one of the excised rat tissue only to maintain homogeneity with the gut tissue sample (control). The experiments were carried out in concordance with the Danish law on animal experiments as approved by the Danish Animal Experiments Inspectorate in accordance with the EU directive. Since the aim of the study was to verify TRAP microdevices activation in vivo, and subsequent entrapment of gut microbiota in the hydrogel matrix, excised animal tissue and mucus layer served as a control. We also delivered oral insulin $(1.1 \mathrm{mg})$ but no relevant biological response was observed.

Histology: Portion of rat intestine was excised and stored at $-20 \mathrm{C}$. A section of the tissue was cut with a scalpel and placed in $4 \%$ formaldehyde solution (BioSafe $®$ ). The sections were H\&E stained according to standard operation procedure. Note that microvilli structures were not observed due to the autolytic degradation.

\section{Proteolytic enzyme activity in TRAP bots (post-in vivo administration)}

\section{Material preparation}

Pierce $^{\mathrm{TM}}$ Protease Assay Kit was obtained from Thermo Scientific, Denmark. $50 \mathrm{mM}$, $\mathrm{pH} 8.5$ assay buffer was prepared by dissolving the contents of BupH Borate Pack in $500 \mathrm{~mL}$ distilled water. Lyophilized Succinylated Casein was dissolved in assay buffer to get $2 \mathrm{mg} / \mathrm{mL}$ Succinylated Casein solution. Lyophilized TPCK Trypsin was then dissolved in assay buffer to prepare a $50 \mathrm{mg} / \mathrm{mL}$ stock solution. Sample was stored at $-80{ }^{\circ} \mathrm{C}$. TNBSA stock was diluted in assay buffer to make TNBSA working solution.

\section{Standard curve of Trypsin activity}

Trypsin stock solution was diluted to $0.5 \mathrm{mg} / \mathrm{mL}$ in Assay buffer. The stock solution was used to prepare serial dilutions of $0.1,0.2,0.3,0.4,0.5 \mathrm{mg} / \mathrm{mL} .100 \mu \mathrm{L}$ Assay 
Buffer was added into the first well in 96-well plate (TS Varioskan Multiplate reader), serving as a blank control. $100 \mu \mathrm{L}$ Succinylated Casein Solution was pipetted into the next five wells, followed by the addition of $50 \mu \mathrm{L}$ serial dilutions in proper order. The plate was incubated for 20 minutes at $37^{\circ} \mathrm{C}$ in the drying oven. Then $50 \mu \mathrm{L}$ of TNBSA Working Solution was added into each well and the plate was incubated for another 20 minutes at room temperature. The change in Trypsin activity was detected by measuring the absorbance at $450 \mathrm{~nm}\left(\triangle \mathrm{A}_{450}\right)$ with constant shaking (pulsed) at 600 rpm. A standard curve was plotted.

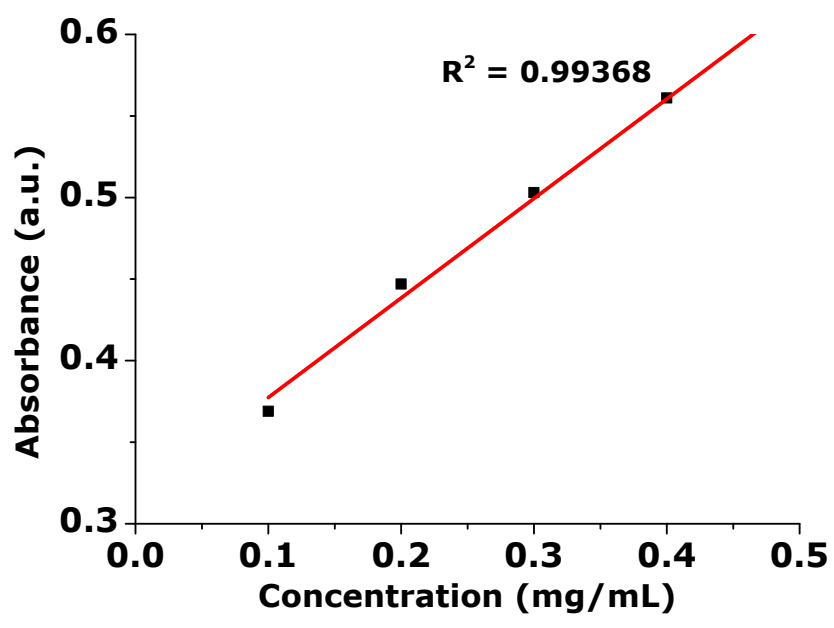

\section{Proteolytic activity assessment in TRAP bots}

TRAP bots were recovered from the ileum tissue and washed with acetate buffer $(\mathrm{pH}$ 4) to remove adhered mucus, followed by PBS, and distilled water, each washing was done three times (i.e. a total of nine washes). The cleaned TRAP bots were ready for the trypsin activity testing. The study was performed using the same method as is described for trypsin activity standard curve, except instead of the serial dilutions, 50 $\mu \mathrm{L}$ Assay Buffer containing an increasing number of TRAP bots $(1,3,5,7,9)$ was added. The well with no TRAP bots was set as blank control. A duplicated set of wells was prepared and the absorbance at $450 \mathrm{~nm}$ wavelength $\left(\Delta \mathrm{A}_{450}\right)$ was measured with constant shaking (pulsed) at $600 \mathrm{rpm}$.

\section{Sample preparation for qPCR sequencing}

Sample 1 (Scrapped mucus with TRAPs): Mucus layer from the excised tissue was gently scrapped off with a stainless steel spatula together with TRAP microdevices. This sample was kept in an Eppendorf tube and stored at $-20{ }^{\circ} \mathrm{C}$ until further use. Sample 2 (multiwash-TRAPs): TRAP bots were taken from the ileum tissue and washed thrice, each with acetate buffer (pH 4), PBS, 70\% EtOH, 2 minutes each with 
vigorous vortex - i.e. a total of nine washes for each microdevice. Sample 3 (PBSwash TRAPs) were prepared the same way as above but only utilized PBS for washing (thrice), two minutes each cycle with vigorous vortex. The DNA extraction from the TRAP microdevices were performed by using the PureLink TM Microbiome DNA Purification Kit, which include beatbeading. The sequencing library was prepared using the standard Illumina MiSeq protocol for amplicon sequencing, using the V3-V4 primer regions. The regions that were amplified in the primary PCR, using the 341F-805R primers for the V3-V4 16S rRNA gene regions. The raw sequencing reads were run through the standard BION pipeline and mapped with the RDP reference database (James E. McDonald, 2016, Characterising the Canine Oral Microbiome by Direct Sequencing of Reverse-Transcribed rRNA Molecules - supplement materials 1). Dataanalysis of the 16S rRNA gene sequencing data was performed in R (v. 3.6), using the packages: Vegan (v. 2.6-5), Ampvis2 (v. 2.6.2) and Phyloseq (v.1.16.2) Reads not belonging to bacteria were filtrated from the dataset. The cut off limit is $0.1 \%$, meaning that genera present in lower numbers than $0.1 \%$ are not included.

\begin{tabular}{|l|c|c|c|}
\hline \multicolumn{1}{|c|}{ Sample name } & $\begin{array}{c}\text { Quibit, } \\
\mathbf{n g} / \boldsymbol{\mu l}\end{array}$ & $\begin{array}{c}\text { Frag analyser, } \\
\mathbf{n g} / \mathbf{\mu l}\end{array}$ & $\begin{array}{c}\text { Frag analyser, } \\
\mathbf{n m o l} / \mathbf{l}\end{array}$ \\
\hline Sample 2 (TRAPs with mucus) & 11,2 & 10,0 & 28,6 \\
\hline Sample 3 (TRAPs multi wash) & 9,6 & 10,3 & 27,7 \\
\hline Sample 4 (TRAPs PBS wash only) & 14,6 & 12,1 & 33,4 \\
\hline
\end{tabular}

Table S1. Secondary index pcr measurement on the fragment analyzer and qubit.
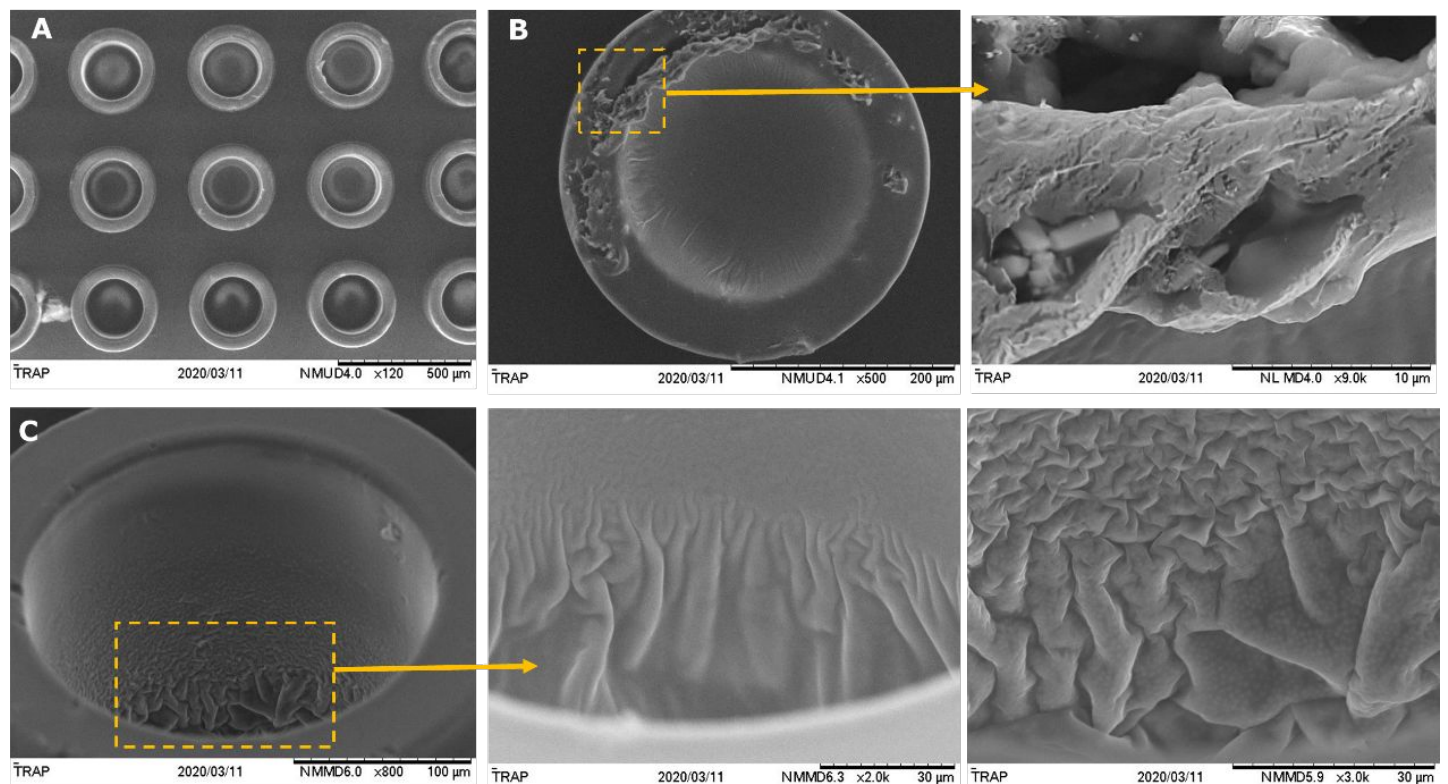
Figure S1 SEM images of TRAP microdevices after activation: A) Homogeneous activation of multiple TRAP microdevices; B) Hydrogel formation in one of the activated TRAP microdevice. Inset highlights gelation onto the rim of the microdevice too; C) A TRAP microdevice with PEGDA polymerization along the inner cavity of the reaction microchamber (possibly due to partial loss of PEGDA monomer). Inset highlights polymerized PEGDA gel topography. Original scale bar included.

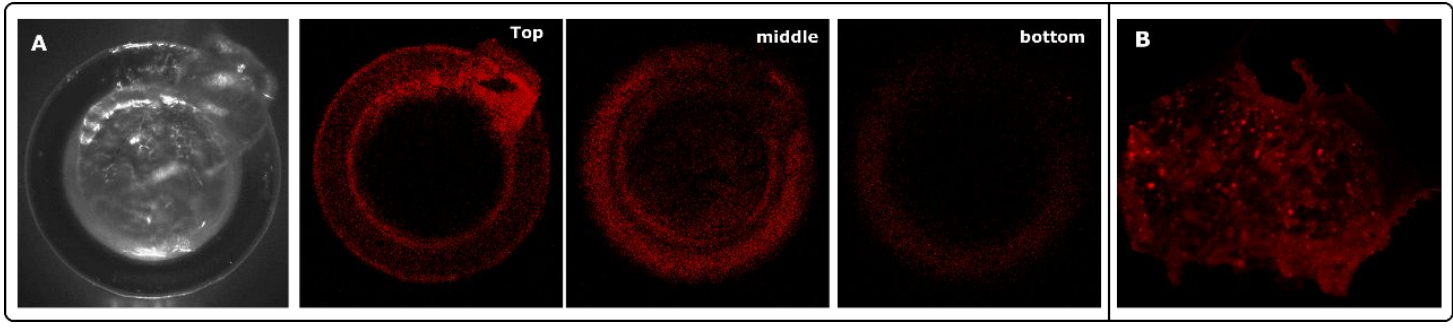

Figure S2 Confocal fluorescence microscope image of a TRAP microdevice when activated in the presence of E.coli DH5a bacteria stained with Bactlight ${ }^{\circledR}$ dye (Abs/Em: 555/580 nm): A) Brightfield image and associated Z-stack images of the same microdevice depicting different regions: top, mid, bottom. B) Fluorescence image (red) of bacteria-enmeshed hydrogel.

\section{TRAPs in the gut}
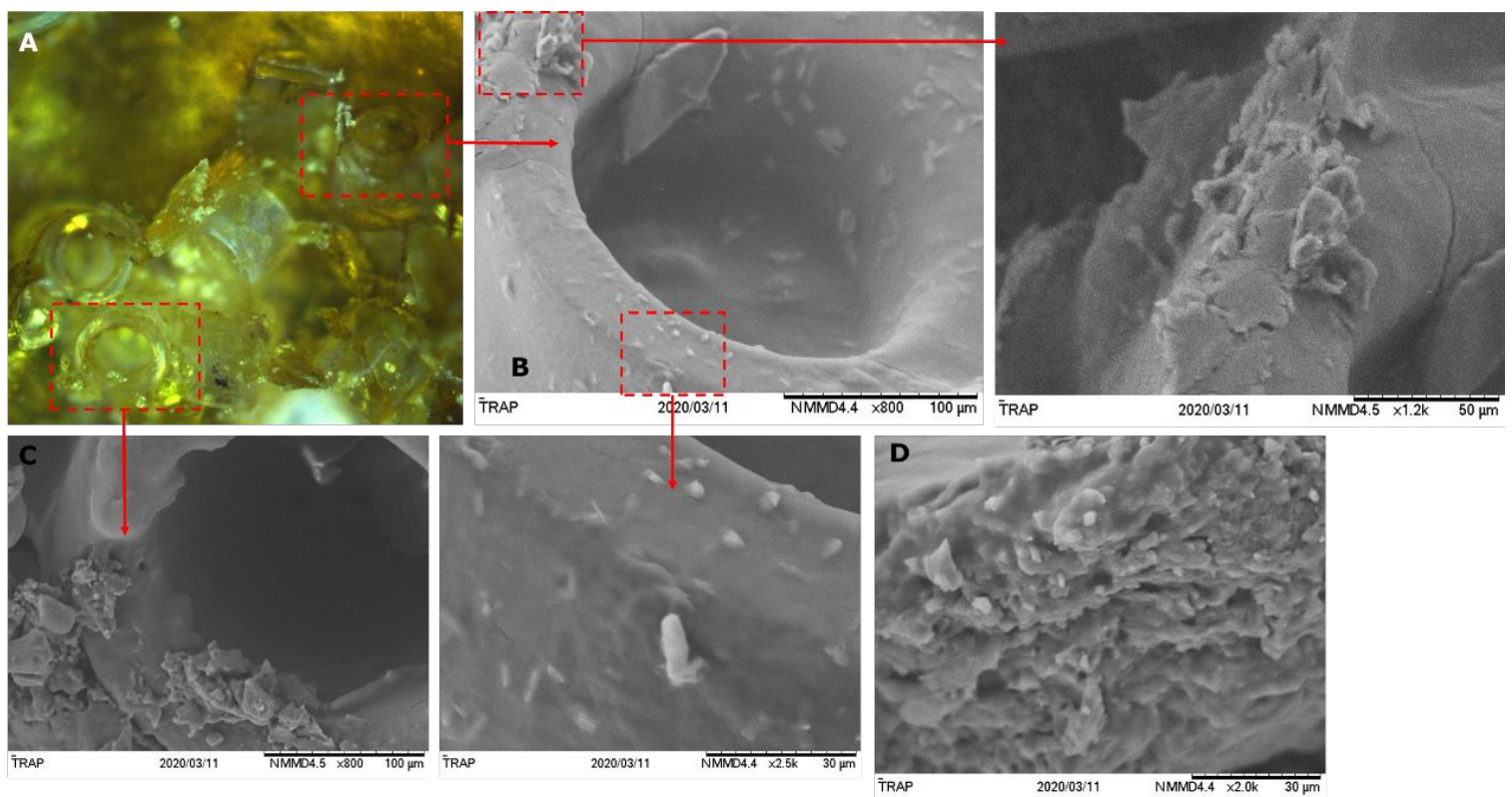

Figure S3. Images of excited gut tissue with TRAP microdevices. A) Optical image; B) SEM image of highlighted region from $A$ depicting entrapped bacteria; C) SEM image from highlighted regions of $A$ depicting polymerized PEGDA inside the microdevice; D) PEGDA hydrogel with enmeshed bacteria. (SEM images were obtained with a $15 \mathrm{~nm}$ sputtered gold layer). Original scale bar included. 


\section{Biomarker quantification}

We demonstrated detection and quantification of proteolytic enzyme (trypsin) as a model biomarker. Trypsin cleaves a succinylated casein substrate to generate peptide fragments with free amino-terminal groups. These peptides reacted with 2,4,6trinitrobenzenesulfonic acid (TNBSA), followed by the measurement of the absorbance increase that results from the formation of yellow colored TNB-peptide adducts $\left(\lambda_{\max }=\right.$ $405 \mathrm{~nm}$ ). As expected, trypsin activity gradually increased with increasing number of TRAP microdevices as shown in Figure S1. Nonetheless, decrease in enzymatic activity upon increasing the number of TRAP microdevices was observed which can be attributed to dimerization of proteolytic enzymes as well as stearic hindrances owing to the presence of high amount of enzymes in a small reaction volume (200 $\mu \mathrm{L})$. In our study, we chose a ubiquitous class of proteolytic enzymes owing to their importance in maintaining gut homeostasis. Increased trypsin activity in the tissue has been linked to Crohn's disease and UC

\section{Control Trypsin assay: TRAP microdevices}

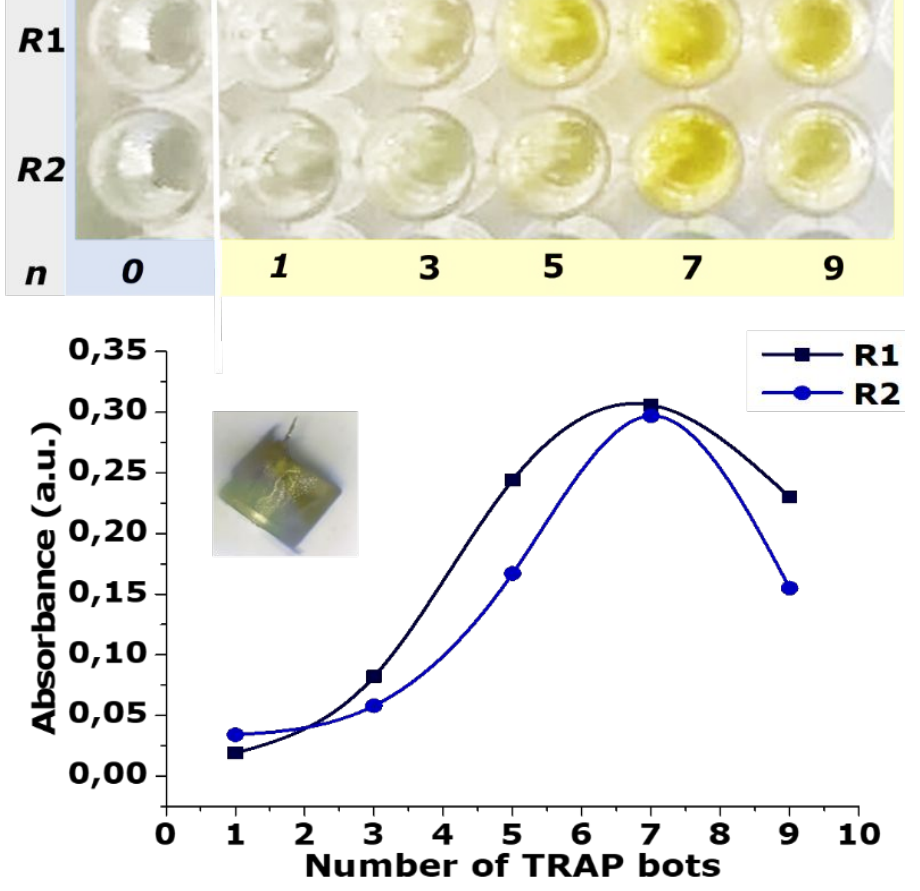

Figure S4. Showing protein biomarker (Trypsin) assessment from excised TRAP microdevices (after oral gavaging). Inset image showing one such isolated microdevice. 


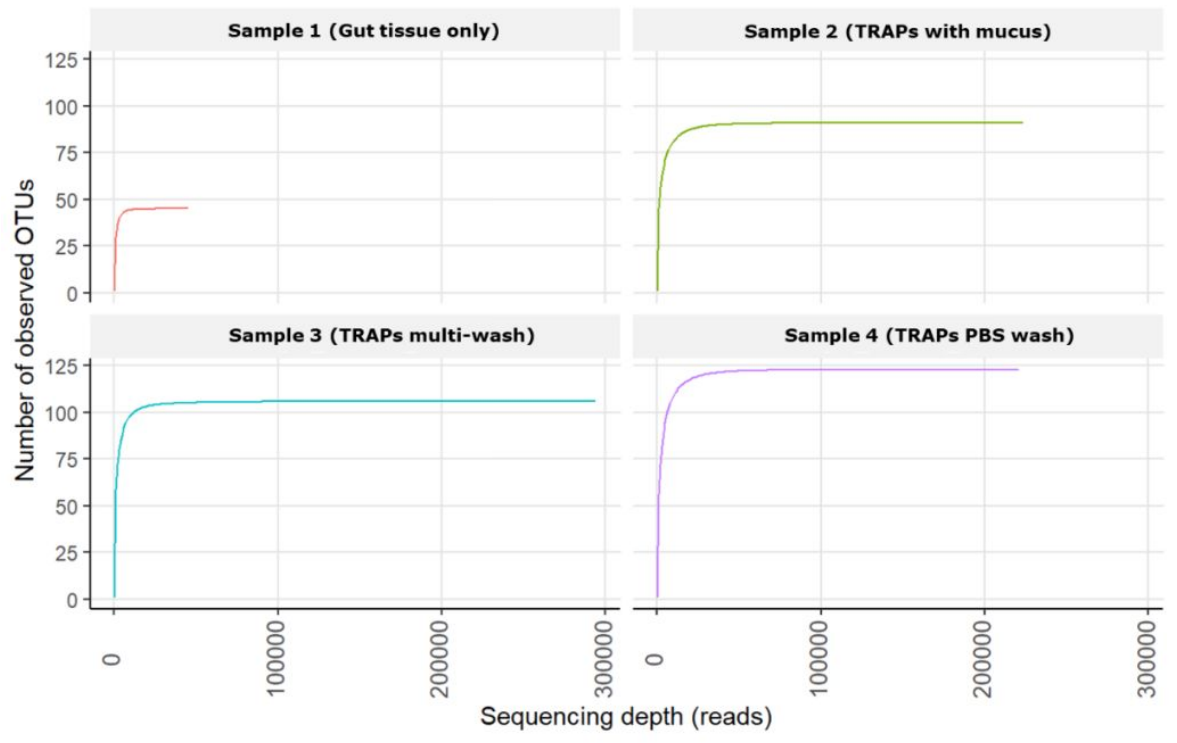

Figure S5: Rarefaction curve at genus level (Sample1: Gut tissue only; Sample 2: TRAPS with scrapped mucus; Sample 3: TRAPs multi-wash; and Sample 4: TRAPs PBS-wash)

\begin{tabular}{|c|c|c|c|c|}
\hline Bacterial genera & Sample 1 & Sample 2 & Sample 3 & Sample 4 \\
\hline \multirow{26}{*}{$\begin{array}{r}\text { Listeria - } \\
\text { Bifidobacterium - } \\
\text { Lactobacillus - } \\
\text { Bacillus - } \\
\text { Romboutsia - } \\
\text { Staphylococcus - } \\
\text { Corynebacterium - } \\
\text { Propionibacterium - } \\
\text { Lactococcus - } \\
\text { Escherichia - } \\
\text { Pseudomonas - } \\
\text { Faecalibaculum - } \\
\text { Enterococcus - } \\
\text { Leuconostoc - } \\
\text { Micrococcus - } \\
\text { Salmonella - } \\
\text { Ralstonia - } \\
\text { Cupriavidus - } \\
\text { Haemophilus - } \\
\text { Peptoniphilus - } \\
\text { Rhizobium - } \\
\text { Leifsonia - } \\
\text { Mobiluncus - } \\
\text { Acinetobacter - } \\
\text { Serratia - }\end{array}$} & 32.2 & 4.1 & 0.5 & 1.9 \\
\hline & 25.9 & 1.7 & 0 & 0 \\
\hline & 10.2 & 12.1 & 5 & 7.3 \\
\hline & 9.8 & 0.3 & 0.4 & 0.2 \\
\hline & 9.2 & 27.3 & 0.2 & 0 \\
\hline & 4.3 & 0.3 & 1.2 & 2.7 \\
\hline & 1.7 & 1.9 & 2.6 & 5.3 \\
\hline & 1.1 & 0.4 & 1.2 & 3.1 \\
\hline & 0.9 & 0.3 & 0.7 & 2.6 \\
\hline & 0.7 & 0 & 0 & 0 \\
\hline & 0.5 & 1.7 & 1.9 & 1.5 \\
\hline & 0.4 & 0.3 & 0 & 0 \\
\hline & 0.3 & 1.7 & 0 & 0.1 \\
\hline & 0.2 & 10.4 & 17.1 & 8.9 \\
\hline & 0.2 & 0.2 & 0.1 & 0.3 \\
\hline & 0.2 & 0 & 0 & 0 \\
\hline & 0.2 & 0.1 & 0.1 & 0 \\
\hline & 0.1 & 0 & 0 & 0 \\
\hline & 0.1 & 0 & 0 & 0.1 \\
\hline & 0.1 & 0.1 & 0.5 & 0.3 \\
\hline & 0.1 & 0.3 & 0.6 & 0.4 \\
\hline & 0.1 & 0 & 0.1 & 0 \\
\hline & 0.1 & 0 & 0 & 0 \\
\hline & 0.1 & 0.2 & 1 & 0.5 \\
\hline & 0.1 & 0.3 & 0.3 & 0 \\
\hline & $\begin{array}{l}\text { Sample } 1 \text { (Gut } \\
\text { tissue only) }\end{array}$ & $\begin{array}{l}\text { Sample } 2 \\
\text { (TRAPs with } \\
\text { mucus) }\end{array}$ & $\begin{array}{c}\text { Sample } 3 \\
\text { (TRAPs multi- } \\
\text { wash) }\end{array}$ & $\begin{array}{l}\text { Sample } 4 \\
\text { (TRAPs PBS } \\
\text { wash only) }\end{array}$ \\
\hline
\end{tabular}

Figure S6: Heatmap of 25 most abundant genera arranged w.r.t. Sample 1 (Gut tissue only). 